LDL-C was reduced from 2.37 to $2.25 \mathrm{mmol} / \mathrm{I}$ (91.7 to $86.8 \mathrm{mg} / \mathrm{dl} ; P=0.001$ ).

In phase two, patients were randomized to either continue with pharmacy care for 6 months, or return to their usual care. On final assessment, mean medication adherence was 95.5\% for the group continuing to receive pharmacy care, but had dropped to $69.1 \%$ for the group reverting to usual care. Mean systolic BP decreased by a further $5.9 \mathrm{mmHg}$ in the pharmacy care group $(P=0.04)$, but there were no further significant differences in LDL-C levels.

Original article Lee JK et al. (2006) Effect of a pharmacy care program on medication adherence and persistence, blood pressure, and low-density lipoprotein cholesterol. JAMA 296: 2563-2571

\section{Long-term follow-up needed after bone marrow mononuclear cell therapy for limb ischemia}

Therapeutic options for critical limb ischemia are limited, and patients are often unresponsive to drug therapy or unsuitable for percutaneous or surgical revascularization. Transplantation of bone marrow mononuclear cells has previously been shown to have short-term clinical benefits for these 'no option' patients. Miyamoto et al. report on an uncontrolled, unblinded pilot study of the long-term safety and efficacy of transplantation therapy in patients with thromboangiitis obliterans.

Cell transplantations were performed in 11 limbs of 8 patients. At 4 weeks, improvements in pain, as measured by a visual analog scale, were observed in all 11 limbs; 4 limbs were reported to have complete pain relief. Skin uclers improved in all eight limbs with baseline ischemic ulcers, with complete healing seen in all but one. Long-term follow-up was in the range 103-1,466 days (mean \pm SD $684 \pm 549$ days), during which adverse events (including sudden death and unfavorable angiogenesis) were observed in 4 of 8 patients. As the prognosis is usually good for patients with thromboangiitis obliterans who stop smoking, the authors note that these adverse events might have been related to the transplantation procedure-possibly via accelerated atherogenesis, or unregulated cell differentiation and proliferation.

Miyamoto et al. conclude that sufficient knowledge regarding the safety and efficacy of bone marrow mononuclear cell transplantation for patients with critical limb ischemia is lacking. Careful long-term monitoring will consequently be required for any patients undergoing this procedure.

Original article Miyamoto K et al. (2006) Unblinded pilot study of autologous transplantation of bone marrow mononuclear cells in patients with thromboangiitis obliterans. Circulation 114: 2679-2684

\section{Increased incidence of thoracic aortic disease, but improved postoperative survival}

Knowledge regarding the prevalence of thoracic aortic diseases (TAD) comes almost entirely from small studies, many of which predate the advent of modern diagnostic procedures such as CT and echocardiography. In a nationwide population-based study in Sweden that encompassed 14,000 cases of thoracic aortic aneurysm or dissection from 1987 to 2002, Olsson et al. found that the incidence of TAD was higher than previously reported.

Incidence in men increased from 10.7 per 100,000 /year in 1987 to 16.3 per 100,000/year in 2002; incidence in women increased from 7.1 to 9.1 per $100,000 /$ year over the same period. Operations for TAD increased sevenfold in men, from 0.8 to 5.6 per 100,000/year, and 15 -fold in women, from 0.2 to 3.0 per $100,000 / y e a r$. Surgery-related 30 -day mortality decreased from $25 \%$ to $13 \%$ over the study period, and risk of death decreased with later year of operation (hazard ratio 0.67 for 1999-2002 compared with 1987-1990). Age $\geq 60$ years was independently associated with long-term mortality. Post-operative long-term survival was identical whether patients were operated on for aortic aneurysms, dissections or ruptures. Overall survival was better in patients who underwent surgery than in those who did not.

The authors suggest that the increased incidence of TAD is caused largely by the improved diagnostic procedures now in use. In view of the survival benefit obtained by patients treated with surgery, the authors recommend early surgery whenever possible.

Original article Olsson C et al. (2006) Thoracic aortic aneurysm and dissection: increasing prevalence and improved outcomes reported in a nationwide populationbased study of more than 14,000 cases from 1987 to 2002 . Circulation 114: 2611-2618 